\title{
STRONGLY NONLINEAR POTENTIAL THEORY ON METRIC SPACES
}

\author{
NOUREDDINE AÏSSAOUI
}

Received 23 January 2002

We define Orlicz-Sobolev spaces on an arbitrary metric space with a Borel regular outer measure, and we develop a capacity theory based on these spaces. We study basic properties of capacity and several convergence results. We prove that each Orlicz-Sobolev function has a quasi-continuous representative. We give estimates for the capacity of balls when the measure is doubling. Under additional regularity assumption on the measure, we establish some relations between capacity and Hausdorff measures.

\section{Introduction}

The introduction and the extensive study of Sobolev spaces on arbitrary metric spaces by Franchi et al. [9], Hajłasz [12], Hajłasz and Koskela [13], Hajłasz and Martio [14], and others, have given a great impulse to several developments in geometric analysis on metric measure spaces. Important examples are the substantial progress of various domains such as fractals, partial differential equations, Carnot-Carathéodory geometries, stochastic process, and so forth.

The nonlinear potential theory on metric spaces has seen a great jump since the development of the capacity theory in these spaces by Kilpeläinen et al. [16], Kinnunen and Martio [17], and others.

For Orlicz and Orlicz-Sobolev spaces in the Euclidean space, we have developed in $[2,3,4,5,6,7]$ a potential theory, called strongly nonlinear potential theory. It is natural to develop this theory in the setting of metric spaces. It is the object of this paper.

A Lipschitz characterization of Orlicz-Sobolev spaces in Euclidean case is given. Since a density argument and the Hardy-Littlewood maximal function are involved, we must suppose the Orlicz space to be reflexive. This characterization is used to introduce a definition of Orlicz-Sobolev spaces on an arbitrary metric measure space. We prove an approximation theorem of Orlicz-Sobolev 
functions by Lipschitz functions, both in Lusin and in norm sense. This generalizes a result by Hajłasz in [12] relative to the Sobolev case.

Then we develop a capacity theory based on the definition of Orlicz-Sobolev spaces on an arbitrary metric measure space. Basic properties of capacity and several convergence results are studied. Moreover, we prove that each OrliczSobolev function has a quasi-continuous representative and we give estimates for the capacity of balls when the measure is doubling. Some relations between capacity and Hausdorff measures are established under additional regularity assumption on the measure.

This paper is organized as follows. In Section 2, we list the prerequisites from the Orlicz theory. Section 3 is dedicated to Lipschitz characterization of OrliczSobolev spaces in the Euclidean case, to the study of Orlicz-Sobolev spaces on metric spaces and to establish an approximation theorem of Orlicz-Sobolev functions by Lipschitz functions. Section 4 is reserved to establish important properties of capacity on metric spaces. Section 5 deals with the comparison between capacity and measure in the metric space, and between capacity and the Hausdorff measure.

\section{Preliminaries}

An $N$-function is a continuous convex and even function $\Phi$ defined on $\mathbb{R}$, verifying $\Phi(t)>0$ for $t>0, \lim _{t \rightarrow 0} \Phi(t) / t=0$, and $\lim _{t \rightarrow+\infty} \Phi(t) / t=+\infty$.

We have the representation $\Phi(t)=\int_{0}^{|t|} \varphi(x) d \mathfrak{L}(x)$, where $\varphi: \mathbb{R}^{+} \rightarrow \mathbb{R}^{+}$is nondecreasing, right continuous, with $\varphi(0)=0, \varphi(t)>0$ for $t>0, \lim _{t \rightarrow 0^{+}} \varphi(t)=0$, and $\lim _{t \rightarrow+\infty} \varphi(t)=+\infty$. Here $\mathfrak{L}$ stands for the Lebesgue measure. We put in the sequel, as usual, $d x=d \mathfrak{L}(x)$.

The $N$-function $\Phi^{*}$ conjugate to $\Phi$ is defined by $\Phi^{*}(t)=\int_{0}^{|t|} \varphi^{*}(x) d x$, where $\varphi^{*}$ is given by $\varphi^{*}(s)=\sup \{t: \varphi(t) \leq s\}$.

Let $\Phi$ be an $N$-function. We say that $\Phi$ verifies the $\Delta_{2}$ condition if there is a constant $C>0$ such that $\Phi(2 t) \leq C \Phi(t)$ for all $t \geq 0$. We denote by $C(\Phi)$ the smallest of such constants. That is, $C(\Phi)=\sup _{t>0} \Phi(2 t) / \Phi(t)$.

The $\Delta_{2}$ condition for $\Phi$ can be formulated in the following equivalent way: for every $C>0$ there exists $C^{\prime}>0$ such that $\Phi(C t) \leq C^{\prime} \Phi(t)$ for all $t \geq 0$.

Let $(X, \Gamma, \mu)$ be a measure space and $\Phi$ an $N$-function. The Orlicz class $\mathscr{L}_{\Phi, \mu}(X)$ is defined by

$$
\mathscr{L}_{\Phi, \mu}(X)=\left\{f: X \rightarrow \mathbb{R} \text { measurable }: \int_{X} \Phi(f(x)) d \mu(x)<\infty\right\}
$$

We define the Orlicz space $\mathbf{L}_{\Phi, \mu}(X)$ by

$$
\mathbf{L}_{\Phi, \mu}(X)=\left\{f: X \longrightarrow \mathbb{R} \text { measurable }: \int_{X} \Phi(\alpha f(x)) d \mu(x)<\infty \text { for some } \alpha>0\right\}
$$


We always have $\mathscr{L}_{\Phi, \mu}(X) \subset \mathbf{L}_{\Phi, \mu}(X)$. The equality $\mathscr{L}_{\Phi, \mu}(X)=\mathbf{L}_{\Phi, \mu}(X)$ occurs if $\Phi$ verifies the $\Delta_{2}$ condition.

The Orlicz space $\mathbf{L}_{\Phi, \mu}(X)$ is a Banach space with the following norm, called the Luxemburg norm,

$$
\|\mid f\|_{\Phi, \mu, X}=\inf \left\{r>0: \int_{X} \Phi\left(\frac{f(x)}{r}\right) d \mu(x) \leq 1\right\} .
$$

Recall that $\mathbf{L}_{\Phi, \mu}(X)$ is reflexive if $\Phi$ and $\Phi^{*}$ verify the $\Delta_{2}$ condition.

Note that if $\Phi$ verifies the $\Delta_{2}$ condition, then $\int \Phi\left(f_{i}(x)\right) d \mu \rightarrow 0$ as $i \rightarrow \infty$ if and only if $\left\|\left|f_{i}\right|\right\|_{\Phi, \mu, X} \rightarrow 0$ as $i \rightarrow \infty$.

Let $\Omega$ be an open set in $\mathbb{R}^{N}, \mathbf{C}^{\infty}(\Omega)$ be the space of functions which, together with all their partial derivatives of any order, are continuous on $\Omega$, and $\mathrm{C}_{0}^{\infty}\left(\mathbb{R}^{N}\right)=\mathbf{C}_{0}^{\infty}$ stands for all functions in $\mathbf{C}^{\infty}\left(\mathbb{R}^{N}\right)$ which have compact support in $\mathbb{R}^{N}$. The space $\mathrm{C}^{k}(\Omega)$ stands for the space of functions having all derivatives of order $\leq k$ continuous on $\Omega$, and $\mathrm{C}(\Omega)$ is the space of continuous functions on $\Omega$.

The (weak) partial derivative of $f$ of order $|\beta|$ is denoted by

$$
D^{\beta} f=\frac{\partial^{|\beta|}}{\partial x_{1}^{\beta_{1}} \cdot \partial x_{2}^{\beta_{2}} \cdots \partial x_{N}^{\beta_{N}}} f .
$$

Let $\Phi$ be an $N$-function and $m \in \mathbb{N}$. We say that a function $f: \mathbb{R}^{N} \rightarrow \mathbb{R}$ has a distributional (weak partial) derivative of order $m$, denoted by $D^{\beta} f,|\beta|=m$, if

$$
\int f D^{\beta} \theta d x=(-1)^{|\beta|} \int\left(D^{\beta} f\right) \theta d x, \quad \forall \theta \in \mathbf{C}_{0}^{\infty} .
$$

Let $\Omega$ be an open set in $\mathbb{R}^{N}$ and denote $\mathbf{L}_{\Phi, \mathfrak{L}}(\Omega)$ by $\mathbf{L}_{\Phi}(\Omega)$. The Orlicz-Sobolev space $W^{m} \mathbf{L}_{\Phi}(\Omega)$ is the space of real functions $f$, such that $f$ and its distributional derivatives up to the order $m$, are in $\mathbf{L}_{\Phi}(\Omega)$.

The space $W^{m} \mathbf{L}_{\Phi}(\Omega)$ is a Banach space equipped with the norm

$$
\||f|\|_{m, \Phi}=\sum_{0 \leq|\beta| \leq m}\left\|\left|D^{\beta} f\right|\right\|_{\Phi}, \quad f \in W^{m} \mathbf{L}_{\Phi}(\Omega),
$$

where $\left\|\left|D^{\beta} f\right|\right\|_{\Phi}=\left\|\left|D^{\beta} f\right|\right\|_{\Phi, \mathfrak{L}, \Omega}$.

Recall that if $\Phi$ verifies the $\Delta_{2}$ condition, then $\mathbf{C}^{\infty}(\Omega) \cap W^{m} \mathbf{L}_{\Phi}(\Omega)$ is dense in $W^{m} \mathbf{L}_{\Phi}(\Omega)$, and $\mathbf{C}_{0}^{\infty}\left(\mathbb{R}^{N}\right)$ is dense in $W^{m} \mathbf{L}_{\Phi}\left(\mathbb{R}^{N}\right)$.

For more details on the theory of Orlicz spaces, see $[1,18,19,20,21]$.

\section{Orlicz-Sobolev spaces on metric spaces}

3.1. The Euclidean case. We begin by two lemmas which lead to a Lipschitz characterization of Orlicz-Sobolev spaces. 
Lemma 3.1. Let $\Phi$ be an $N$-function satisfying the $\Delta_{2}$ condition, $Q$ a cube in $\mathbb{R}^{N}$, and $f \in W^{1} \mathbf{L}_{\Phi}(Q)$. Then, for almost all $x \in Q$,

$$
\left|f(x)-f_{Q}\right| \leq C \int_{Q} \frac{|\nabla f(y)|}{|x-y|^{N-1}} d y,
$$

where $f_{Q}=1 / \mu(Q) \int_{Q} f d \mu$, and the constant $C$ depends only on $N$ and $Q$.

Proof. It is enough to establish (3.1) for $\mathbf{C}^{1}(Q)$. But in this case the proof can be found in [11, Lemma 7.16].

We omit the proof of the following lemma (Hedberg's inequality) since it is exactly the same as the one in [15] or in [23, Lemma 2.8.3].

Lemma 3.2. Let $\Phi$ be an $N$-function and $f \in W^{1} \mathbf{L}_{\Phi}\left(\mathbb{R}^{N}\right)$. Then there is a constant $C$ depending only on $N$ such that for all $x \in \mathbb{R}^{N}$,

$$
\int_{B(x, R)} \frac{|\nabla f(y)|}{|x-y|^{N-1}} d y \leq C R M_{R}(|\nabla f|)(x),
$$

where $M_{R}(h)(x)=\sup _{r<R}(1 /|B(x, r)|) \int_{B(x, r)}|h(y)| d y$ and $|B(x, r)|$ is the Lebesgue measure of the ball $B(x, r)$ on $\mathbb{R}^{N}$.

Now we give a Lipschitz characterization of Orlicz-Sobolev spaces.

Theorem 3.3. Let $\Phi$ be an $N$-function such that $\Phi$ and $\Phi^{*}$ satisfy the $\Delta_{2}$ condition. Then $f \in W^{1} \mathbf{L}_{\Phi}\left(\mathbb{R}^{N}\right)$ if and only if there exists a nonnegative function $g \in \mathbf{L}_{\Phi}\left(\mathbb{R}^{N}\right)$ such that

$$
|f(x)-f(y)| \leq|x-y|[g(x)+g(y)]
$$

for all $x, y \in \mathbb{R}^{N} \backslash F,|F|=0$.

Proof. Let $Q$ be a cube in $\mathbb{R}^{N}$ and $x, y \in Q$. Then we can find a subcube $Q^{*}$ with $x, y \in Q^{*}$ and $\operatorname{diam} Q^{*} \approx|x-y|$. Here $A \approx B$ if there is a constant $C$ such that $C^{-1} \leq A \leq C B$. Let $f \in W^{1} \mathbf{L}_{\Phi}\left(\mathbb{R}^{N}\right)$. Then by (3.1) and (3.2) we get

$$
\begin{aligned}
|f(x)-f(y)| & \leq\left|f(x)-f_{\mathrm{Q}^{*}}\right|+\left|f(y)-f_{\mathrm{Q}^{*}}\right| \\
& \leq C|x-y|\left(\mathcal{M}_{|x-y|}(|\nabla f|)(x)+M_{|x-y|}(|\nabla f|)(y)\right) \\
& \leq C|x-y|(M(|\nabla f|)(x)+M(|\nabla f|)(y)),
\end{aligned}
$$

where $M(h)(x)=\sup _{0<r}(1 /|B(x, r)|) \int_{B(x, r)}|h(y)| d y$ is the Hardy-Littlewood maximal function. Since $\Phi^{*}$ verifies the $\Delta_{2}$ condition, by [10] the maximal operator is bounded in $\mathbf{L}_{\Phi}\left(\mathbb{R}^{N}\right)$. Hence, there is a nonnegative function $g \in \mathbf{L}_{\Phi}\left(\mathbb{R}^{N}\right)$ such that

$$
|f(x)-f(y)| \leq|x-y|[g(x)+g(y)] \quad \text { a.e. }
$$


For the reverse implication, it suffices to show that, due to Riesz representation and Radon-Nikodym theorem, there is a nonnegative function $h \in \mathbf{L}_{\Phi}\left(\mathbb{R}^{N}\right)$ such that

$$
\left|\frac{\partial f}{\partial x_{i}}[\phi]\right| \stackrel{\text { def }}{=}\left|-\int f \frac{\partial \phi}{\partial x_{i}} d x\right| \leq \int|\phi| h d x
$$

for all $\phi \in \mathbf{C}_{0}^{\infty}$. Now, integrating (3.3) twice over a ball $B(x, \varepsilon)$, we get

$$
\frac{1}{|B(x, \varepsilon)|} \int_{B(x, \varepsilon)}\left|f(x)-f_{Q^{*}}\right| d x \leq C \varepsilon \frac{1}{|B(x, \varepsilon)|} \int_{B(x, \varepsilon)} g(x) d x .
$$

Let $\psi \in \mathbf{C}_{0}^{\infty}(B(0,1))$ be such that $\int \psi(x) d x=1$. Put $\psi_{\varepsilon}(x)=\varepsilon^{-N} \psi(x / \varepsilon)$. We have

$$
\int f \frac{\partial \phi}{\partial x_{i}} d x=\lim _{\varepsilon \rightarrow 0} \int \frac{\partial \phi}{\partial x_{i}}\left(\psi_{\varepsilon} * f\right)(x) d x=-\lim _{\varepsilon \rightarrow 0} \int \phi(x)\left(\frac{\partial \psi_{\varepsilon}}{\partial x_{i}} * f\right)(x) d x .
$$

Since $\int\left(\partial \psi_{\varepsilon} / \partial x_{i}\right)(x) d x=0$, we get

$$
\frac{\partial \psi_{\varepsilon}}{\partial x_{i}} * f=\left(f-f_{B(x, \varepsilon)}\right) * \frac{\partial \psi_{\varepsilon}}{\partial x_{i}}
$$

Hence

$$
\begin{aligned}
\left|\frac{\partial \psi_{\varepsilon}}{\partial x_{i}} * f\right|(x) & \leq C \varepsilon^{-N-1} \int_{B(x, \varepsilon)}\left|f-f_{B(x, \varepsilon)}\right|(x) d x \\
& \leq C \frac{1}{|B(x, \varepsilon)|} \int_{B(x, \varepsilon)} g(x) d x .
\end{aligned}
$$

Thus

$$
\left|\int f \frac{\partial \phi}{\partial x_{i}} d x\right| \leq \int|\phi| M(g)(x) d x
$$

Now by [10], $\mathcal{M}(g) \in \mathbf{L}_{\Phi}\left(\mathbb{R}^{N}\right)$ since $\Phi^{*}$ satisfies the $\Delta_{2}$ condition. The proof is complete.

3.2. The metric case. Let $(X, d)$ be a metric space and let $\mu$ be a nonnegative Borel regular outer measure on $X$. The triplet $(X, d, \mu)$ will be fixed in the sequel and will be denoted by $X$.

Let $u: X \rightarrow[-\infty,+\infty]$ be a $\mu$-measurable function defined on $X$. We denote by $D(u)$ the set of all $\mu$-measurable functions $g: X \rightarrow[0,+\infty]$ such that

$$
|u(x)-u(y)| \leq d(x, y)(g(x)+g(y))
$$

for every $x, y \in X \backslash F, x \neq y$, with $\mu(F)=0$. The set $F$ is called the exceptional set for $g$. 
Note that the right-hand side of (3.12) is always defined for $x \neq y$. For the points $x, y \in X, x \neq y$ such that the left-hand side of (3.12) is undefined, we may assume that the left-hand side is $+\infty$.

Let $\Phi$ be an $N$-function. The Dirichlet-Orlicz space $\mathbf{L}_{\Phi, \mu}^{1}(X)$ is the space of all $\mu$-measurable functions $u$ such that $D(u) \cap \mathbf{L}_{\Phi, \mu}(X) \neq \varnothing$. This space is equipped with the seminorm

$$
\|\left.|u|\right|_{\mathbf{L}_{\Phi, \mu}^{1}(X)}=\inf \left\{\||g|\|_{\Phi, \mu, X}: g \in D(u) \cap \mathbf{L}_{\Phi, \mu}(X)\right\}
$$

The Orlicz-Sobolev space is $M_{\Phi, \mu}^{1}(X)=\mathbf{L}_{\Phi, \mu}(X) \cap \mathbf{L}_{\Phi, \mu}^{1}(X)$ equipped with the norm

$$
\left\|\left.|u|\right|_{M_{\Phi, \mu}^{1}(X)}=\right\||u|\left\|_{\Phi, \mu, X}+\right\||u| \|_{\mathbf{L}_{\Phi, \mu}^{1}(X)}
$$

Lemma 3.4. Let $g_{1} \in D\left(u_{1}\right), g_{2} \in D\left(u_{2}\right)$, and $\alpha, \beta \in \mathbb{R}$. If $g \geq|\alpha| g_{1}+|\beta| g_{2}$ almost everywhere, then $g \in D\left(\alpha u_{1}+\beta u_{2}\right)$.

Proof. The proof is a simple verification.

Lemma 3.5. Let $\Phi$ be an $N$-function. Let $\left(u_{i}\right)_{i}$ and $\left(g_{i}\right)_{i}$ be two sequences of functions such that, for all $i, g_{i} \in D\left(u_{i}\right)$. If $u_{i} \rightarrow u$ in $\mathbf{L}_{\Phi, \mu}(X)$ and $g_{i} \rightarrow g$ in $\mathbf{L}_{\Phi, \mu}(X)$, then $g \in D(u)$.

Proof. There are two subsequences of $\left(u_{i}\right)_{i}$ and $\left(g_{i}\right)_{i}$, which we denote again by $\left(u_{i}\right)_{i}$ and $\left(g_{i}\right)_{i}$, such that $u_{i} \rightarrow u$ and $g_{i} \rightarrow g, \mu$-a.e. Let, for $i=1,2, \ldots, F_{i}$ be the exceptional set for $g_{i}$ and let $G$ be a set of measure zero such that $u_{i} \rightarrow u$ and $g_{i} \rightarrow$ $g$ on ${ }^{c} G$. We set $F=G \cup\left(\bigcup_{i=1}^{\infty} F_{i}\right)$. It is clear that $\mu(F)=0$ and $|u(x)-u(y)| \leq$ $d(x, y)(g(x)+g(y))$, for all $x, y \in X \backslash F$. This implies that $g \in D(u)$. The proof is complete.

Theorem 3.6. Let $\Phi$ be an $N$-function. Then $M_{\Phi, \mu}^{1}(X)$, equipped with the norm defined by (3.14), is a Banach space.

Proof. It is clear that $M_{\Phi, \mu}^{1}(X)$ is a vector space. It remains to prove that $M_{\Phi, \mu}^{1}(X)$ is complete for the norm defined by (3.14). Let $\left(u_{i}\right)_{i}$ be an arbitrary Cauchy sequence in $M_{\Phi, \mu}^{1}(X)$. Taking if necessary a subsequence, we may assume that $\left\|\left|\left(u_{i}-u_{i+1}\right)\right|\right\|_{M_{\Phi, \mu}^{1}(X)} \leq 2^{-i}$. Set $v_{j}=u_{j}-u_{j+1}$. There exists $h_{j} \in D\left(v_{j}\right) \cap \mathbf{L}_{\Phi, \mu}(X)$ such that $\left\|\left|h_{j}\right|\right\|_{M_{\Phi, \mu}^{1}(X)} \leq 2^{-i}$. Let $g_{1} \in D\left(u_{1}\right)$ be arbitrary and set, for $k \geq 2, g_{k}=$ $g_{1}+\sum_{j=1}^{k-1} h_{j}$. From the identity $u_{k}=u_{1}-\sum_{j=1}^{k-1} v_{j}$ and Lemma 3.4, it follows that $g_{k} \in D(u)$, for all $k \in N$. Now $\left(u_{k}\right)_{k}$ and $\left(g_{k}\right)_{k}$ are Cauchy sequences in $\mathbf{L}_{\Phi, \mu}(X)$. Thus there are limit functions $u=\lim u_{k}$ in $\mathbf{L}_{\Phi, \mu}(X)$ and $g=\lim g_{k}$ in $\mathbf{L}_{\Phi, \mu}(X)$. Lemma 3.5 implies that $g \in D(u)$ and therefore the sequence $\left(u_{k}\right)_{k}$ has a limit in $M_{\Phi, \mu}^{1}(X)$. The proof is complete.

The previous results lead to the following characterization of $M_{\Phi, \mu}^{1}(X)$. 
Lemma 3.7. Let $\Phi$ be an $N$-function. The function $u \in M_{\Phi, \mu}^{1}(X)$ if and only if $u \in$ $\mathbf{L}_{\Phi, \mu}(X)$ and there are functions $u_{i} \in \mathbf{L}_{\Phi, \mu}(X), i=1,2, \ldots$, such that $u_{i} \rightarrow u \mu$-a.e. and $g_{i} \in D\left(u_{i}\right) \cap \mathbf{L}_{\Phi, \mu}(X), i=1,2, \ldots$, such that $g_{i} \rightarrow g \mu$-a.e. for some $g \in \mathbf{L}_{\Phi, \mu}(X)$.

Proof. The proof is an immediate consequence of Lemma 3.5 and Theorem 3.6.

Moreover, $M_{\Phi, \mu}^{1}(X)$ satisfies the following lattice property.

Lemma 3.8. Let $\Phi$ be an $N$-function. Let $u_{1}, u_{2} \in M_{\Phi, \mu}^{1}(X)$. If $g_{1} \in D\left(u_{1}\right)$ and $g_{2} \in D\left(u_{2}\right)$, then

(i) $u=\max \left(u_{1}, u_{2}\right) \in M_{\Phi, \mu}^{1}(X)$ and $\max \left(g_{1}, g_{2}\right) \in D(u) \cap \mathbf{L}_{\Phi, \mu}(X)$;

(ii) $v=\min \left(u_{1}, u_{2}\right) \in M_{\Phi, \mu}^{1}(X)$ and $\max \left(g_{1}, g_{2}\right) \in D(v) \cap \mathbf{L}_{\Phi, \mu}(X)$.

Proof. We prove the case (i) only, the proof of (ii) is similar. Let $g=\max \left(g_{1}, g_{2}\right)$ and suppose that $F_{1}$ and $F_{2}$ are the exceptional sets for $u_{1}$ and $u_{2}$ in (3.12), respectively. It is evident that $u, g \in \mathbf{L}_{\Phi, \mu}(X)$. It remains to show that $g \in D(u)$. Let $U=\left\{x \in X \backslash\left(F_{1} \cup F_{2}\right): u_{1}(x) \geq u_{2}(x)\right\}$. Let $x, y \in U$. Then

$$
|u(x)-u(y)|=\left|u_{1}(x)-u_{1}(y)\right| \leq d(x, y)\left(g_{1}(x)+g_{1}(y)\right)
$$

By the same manner we obtain, for $x, y \in X \backslash U$,

$$
|u(x)-u(y)| \leq d(x, y)\left(g_{2}(x)+g_{2}(y)\right)
$$

For the remaining cases, let $x \in U$ and $y \in X \backslash U$. If $u_{1}(x) \geq u_{2}(y)$, then

$$
|u(x)-u(y)|=u_{1}(x)-u_{2}(y) \leq u_{1}(x)-u_{1}(y) \leq d(x, y)\left(g_{1}(x)+g_{1}(y)\right) .
$$

If $u_{1}(x)<u_{2}(y)$, then

$$
|u(x)-u(y)|=-u_{1}(x)+u_{2}(y) \leq-u_{2}(x)+u_{2}(y) \leq d(x, y)\left(g_{2}(x)+g_{2}(y)\right) .
$$

The case $x \in X \backslash U$ and $y \in U$ follows by symmetry. Hence, for all $x, y \in$ $X \backslash\left(F_{1} \cup F_{2}\right)$ with $\mu\left(F_{1} \cup F_{2}\right)=0,|u(x)-u(y)| \leq d(x, y)(g(x)+g(y))$.

Next, we prove the following important Poincaré inequality for OrliczSobolev functions.

Proposition 3.9. Let $\Phi$ be an $N$-function. If $u \in M_{\Phi, \mu}^{1}(X)$ and $E \subset X$ is $\mu$-measurable with $0<\mu(E)<\infty$, then for every $g \in D(u) \cap \mathbf{L}_{\Phi, \mu}(X)$,

$$
\left\|\left|u-u_{E}\right|\right\|_{\mathbf{L}_{\Phi, \mu}^{1}(E)} \leq 2 \operatorname{diam}(E)|||g| \|_{\mathbf{L}_{\Phi, \mu}^{1}(E)},
$$

where $u_{E}=1 / \mu(E) \int_{E} f d \mu$. 
364 Strongly nonlinear potential theory on metric spaces

Proof. Put $C=2 \operatorname{diam}(E)\||g|\|_{\mathbf{L}_{\Phi, \mu}^{1}(E)}$ and remark that

$$
\left(u-u_{E}\right)(x)=\frac{1}{\mu(E)} \int_{E}(u(x)-u(y)) d \mu(y)
$$

By the Jensen inequality

$$
\begin{aligned}
\int_{E} \Phi\left(\frac{u-u_{E}}{C}\right)(x) d \mu(x) & =\int_{E} \Phi\left[\frac{1}{\mu(E)} \int_{E} \frac{u(x)-u(y)}{C} d \mu(y)\right](x) d \mu(x) \\
& \leq \int_{E}\left[\int_{E} \frac{1}{\mu(E)} \Phi\left(\frac{u(x)-u(y)}{C}\right) d \mu(y)\right] d \mu(x)
\end{aligned}
$$

On the other hand, using the definition of $D(u)$ and the convexity of $\Phi$, we get

$$
\begin{aligned}
\Phi\left(\frac{u(x)-u(y)}{C}\right) & \leq \Phi\left(\frac{g(x)-g(y)}{2\|\mid g\|_{\mathbf{L}_{\Phi, \mu}^{1}(E)}}\right) \\
& \leq \frac{1}{2}\left[\Phi\left(\frac{g(x)}{\|g\|_{\mathbf{L}_{\Phi, \mu}^{1}(E)}}\right)+\Phi\left(\frac{g(y)}{\|g\|_{\mathbf{L}_{\Phi, \mu}^{1}(E)}}\right)\right] .
\end{aligned}
$$

Now we use the fact that $\int_{E} \Phi\left(g(x) /\||g|\|_{\mathbf{L}_{\Phi, \mu}^{1}(E)}\right) d \mu(x) \leq 1$ to deduce that

$$
\begin{aligned}
\int_{E} \Phi & \left(\frac{u-u_{E}}{C}\right)(x) d \mu(x) \\
& \leq \frac{1}{2 \mu(E)} \int_{E} \int_{E}\left[\Phi\left(\frac{g(x)}{\|\mid g\|_{\mathbf{L}_{\Phi, \mu}^{1}(E)}}\right)+\Phi\left(\frac{g(y)}{\|g\|_{\mathbf{L}_{\Phi, \mu}^{1}(E)}}\right)\right] d \mu(y) d \mu(x) \\
& \leq \frac{1}{2 \mu(E)} \int_{E}\left[1+\Phi\left(\frac{g(x)}{\|\mid g\|_{\mathbf{L}_{\Phi, \mu}^{1}(E)}}\right) \mu(E)\right] d \mu(x) \leq 1
\end{aligned}
$$

The proof is complete.

Now, we prove an approximation theorem of Orlicz-Sobolev functions by Lipschitz functions, both in Lusin sense and in norm. This generalizes a result in [12] relative to the Sobolev case.

Theorem 3.10. Let $\Phi$ be an $N$-function satisfying the $\Delta_{2}$ condition and $u \in$ $M_{\Phi, \mu}^{1}(X)$. Then for every $\varepsilon>0$, there is a Lipschitz function $h$ such that

(1) $\mu(\{x: u(x) \neq h(x)\})<\varepsilon$;

(2) $\||u-h|\|_{M_{\Phi, \mu}^{1}(X)}<\varepsilon$.

Proof. Let $u \in M_{\Phi, \mu}^{1}(X)$ and let $g$ be taken from the definition of $\||u|\|_{\mathbf{L}_{\Phi, \mu}^{1}(X)}$. Let $X_{n}=\{x \in X:|u(x)| \leq n$ and $g(x) \leq n\}$. Since $u, g \in \mathbf{L}_{\Phi, \mu}(X)$ and $\Phi$ satisfies the $\Delta_{2}$ condition, we conclude that $u, g \in \mathscr{L}_{\Phi \mu}(X)$. This implies that $\lim _{n \rightarrow \infty} \Phi(n) \mu\left({ }^{c} X_{n}\right)$ $=0$, and $\lim _{n \rightarrow \infty} \mu\left({ }^{c} X_{n}\right)=0$ because $\Phi(n) \rightarrow \infty$ when $n \rightarrow \infty$. The restriction $\left.u\right|_{X_{n}}$ 
is Lipschitz with the constant $2 n$ and can be extended to the Lipschitz function $u^{\prime}$ on $X$ with the same constant (see [8, Section 2.10.4] and [22, Theorem 5.1]). We put $u_{n}=\left(\operatorname{sgn} u^{\prime}\right) \min \left(\left|u^{\prime}\right|, n\right)$. It is clear that $u_{n}$ is Lipschitz with the constant $2 n,\left.u_{n}\right|_{X_{n}}=\left.u\right|_{X_{n}},\left|u_{n}\right| \leq n$ and $\mu\left(\left\{x: u(x) \neq u_{n}(x)\right\}\right) \leq \mu\left({ }^{c} X_{n}\right) \stackrel{n \rightarrow \infty}{\longrightarrow} 0$.

We must simply prove that $u_{n} \rightarrow u$ in $M_{\Phi, \mu}^{1}(X)$ when $n \rightarrow \infty$. We have

$$
\begin{aligned}
\int \Phi\left(u-u_{n}\right) d \mu & =\int_{{ }^{X_{n}}} \Phi\left(u-u_{n}\right) d \mu \\
& \leq \int_{{ }^{X_{n}}} \Phi\left(|u|+\left|u_{n}\right|\right) d \mu \\
& \leq \frac{C_{\Phi}}{2}\left[\int_{{ }^{X_{n}}} \Phi(|u|) d \mu+\int_{{ }^{X_{n}}} \Phi\left(\left|u_{n}\right|\right) d \mu\right] \\
& \leq \frac{C_{\Phi}}{2}\left[\int_{{ }^{X_{n}}} \Phi(|u|) d \mu+\Phi(n) \mu\left({ }^{c} X_{n}\right)\right] \stackrel{{ }_{n \rightarrow \infty}}{\longrightarrow} 0 .
\end{aligned}
$$

Since $\Phi$ satisfies the $\Delta_{2}$ condition, $\left\|\left|u-u_{n}\right|\right\|_{\mathbf{L}_{\Phi, \mu}^{1}(X)} \stackrel{n \rightarrow \infty}{\longrightarrow} 0$. It remains to estimate the gradient. Let

$$
g_{n}= \begin{cases}0 & \text { for } x \in X_{n} \\ g(x)+3 n & \text { for } x \in^{c} X_{n}\end{cases}
$$

We have $\left|\left(u-u_{n}\right)(x)-\left(u-u_{n}\right)(y)\right| \leq d(x, y)\left(g_{n}(x)+g_{n}(y)\right)$. Since $\int \Phi\left(g_{n}\right) d \mu=$ $\int_{{ }^{c} X_{n}} \Phi\left(g_{n}\right) d \mu \rightarrow 0$ as $n \rightarrow \infty$, we conclude that $\left\|\left|g_{n}\right|\right\|_{\mathbf{L}_{\Phi, \mu}^{1}(X)} \rightarrow 0$ when $n \rightarrow \infty$ because $\Phi$ satisfies the $\Delta_{2}$ condition. The theorem follows.

\section{Capacity on metric spaces}

Definition 4.1. For a set $E \subset X$, define $C_{\Phi, \mu}(E)$ by

$$
C_{\Phi, \mu}(E)=\inf \left\{\||| u||_{M_{\Phi, \mu}^{1}(X)}: u \in B(E)\right\}
$$

where $B(E)=\left\{u \in M_{\Phi, \mu}^{1}(X): u \geq 1\right.$ on a neighborhood of $\left.E\right\}$.

If $B(E)=\varnothing$, we set $C_{\Phi, \mu}(E)=\infty$. Functions belonging to $B(E)$ are called admissible functions for $E$.

Remark 4.2. In the definition of $C_{\Phi, \mu}(E)$, we can restrict ourselves to those admissible functions $u$ such that $0 \leq u \leq 1$.

Proof. Let $B^{\prime}(E)=\{u \in B(E): 0 \leq u \leq 1\}$, then $B^{\prime}(E) \subset B(E)$ implies that

$$
C_{\Phi, \mu}(E) \leq \inf \left\{\||| u \mid\|_{M_{\Phi, \mu}^{1}(X)}: u \in B^{\prime}(E)\right\}
$$


On the other hand, let $\varepsilon>0$ and take $u \in B(E)$ such that $\||u|\|_{M_{\Phi, u}^{1}(X)}$ $\leq C_{\Phi}(E)+\varepsilon$. Then $v=\max (0, \min (u, 1)) \in B^{\prime}(E)$, and by Lemma 3.8 we get $D(u) \subset D(v)$. Hence,

$$
\inf \left\{\||| u \mid\|_{M_{\Phi, \mu}^{1}(X)}: u \in B^{\prime}(E)\right\} \leq\||v|\|_{M_{\Phi, \mu}^{1}(X)} \leq\||u|\|_{M_{\Phi, \mu}^{1}(X)} \leq C_{\Phi, \mu}(E)+\varepsilon .
$$

This completes the proof.

We define a capacity as an increasing positive set function $C$ given on a $\sigma$ additive class of sets $\Gamma$, which contains compact sets and such that $C(\varnothing)=0$ and $C\left(\bigcup_{i \geq 1} X_{i}\right) \leq \sum_{i \geq 1} C\left(X_{i}\right)$ for $X_{i} \in \Gamma, i=1,2, \ldots C$ is called outer capacity if for every $X \in \Gamma$,

$$
C(X)=\inf \{C(O): O \text { open, } X \subset O\}
$$

THEOREM 4.3. Let $\Phi$ be an $N$-function. The set function $C_{\Phi, \mu}$ is an outer capacity.

Proof. It is obvious that $C_{\Phi, \mu}(\varnothing)=0$ and that $C_{\Phi, \mu}$ is increasing. For countable subadditivity, let $E_{i}, i=1,2, \ldots$ be subsets of $X$ and let $\varepsilon>0$. We may assume that $\sum_{i=1}^{\infty} C_{\Phi, \mu}\left(E_{i}\right)<\infty$. We choose $u_{i} \in B\left(E_{i}\right)$ and $g_{u_{i}} \in D\left(u_{i}\right) \cap \mathbf{L}_{\Phi, \mu}(X)$ so that for $i=1,2, \ldots$,

$$
\left.\left\|\left.\left|u_{i}\right|\right|_{\Phi, \mu, X}+\right\|\left|g_{u_{i}}\right|\right|_{\Phi, \mu, X} \leq C_{\Phi}\left(E_{i}\right)+\varepsilon 2^{-i}
$$

We show that $v=\sup _{i} u_{i}$ is admissible for $\bigcup_{i=1}^{\infty} E_{i}$ and $g=\sup _{i} g_{u_{i}} \in D(v) \cap$ $\mathbf{L}_{\Phi, \mu}(X)$.

Observe that $v, g \in \mathbf{L}_{\Phi, \mu}(X)$. Define $v_{k}=\max _{1 \leq i \leq k} u_{i}$. By Lemma 3.8 the function $g_{v_{k}}=\max _{1 \leq i \leq k} g_{u_{i}} \in D\left(v_{k}\right) \cap \mathbf{L}_{\Phi, \mu}(X)$. Since $v_{k} \rightarrow v \mu$-a.e., Lemma 3.7 gives $v \in M_{\Phi, \mu}^{1}(X)$. Clearly $v \geq 1$ in a neighborhood of $\bigcup_{i=1}^{\infty} E_{i}$. Hence $C_{\Phi, \mu}\left(\bigcup_{i=1}^{\infty} E_{i}\right) \leq$ $\||v|\|_{M_{\Phi, \mu}^{1}(X)}$.

By [6, Lemma 2],

$$
\||v|\|_{M_{\Phi, \mu}^{1}(X)} \leq \sum_{i=1}^{\infty}\left(||\left|u_{i}\right|\left\|_{\Phi, \mu, X}+\right\|\left|g_{u_{i}}\right| \|_{\Phi, \mu, X}\right) \leq \sum_{i=1}^{\infty} C_{\Phi, \mu}\left(E_{i}\right)+\varepsilon .
$$

Since $\varepsilon$ is arbitrary, we deduce that $C_{\Phi, \mu}\left(\bigcup_{i=1}^{\infty} E_{i}\right) \leq \sum_{i=1}^{\infty} C_{\Phi, \mu}\left(E_{i}\right)$. Hence $C_{\Phi, \mu}$ is a capacity.

It remains to prove that $C_{\Phi, \mu}$ is outer, that is,

$$
C_{\Phi, \mu}(E)=\inf \left\{C_{\Phi, \mu}(O): E \subset O, O \text { open }\right\}
$$

By monotonicity, $C_{\Phi, \mu}(E) \leq \inf \left\{C_{\Phi, \mu}(O): E \subset O, O\right.$ open $\}$. For the reverse inequality, let $\varepsilon>0$ and let $u \in B(E)$ be such that $\||u|\|_{M_{\Phi, \mu}^{1}(X)} \leq C_{\Phi, \mu}(E)+\varepsilon$. Since 
$u \in B(E)$, there is an open set $O, E \subset O$, such that $u \geq 1$ on $O$. This implies that $C_{\Phi, \mu}(O) \leq\||u|\|_{M_{\Phi, \mu}^{1}(X)} \leq C_{\Phi, \mu}(E)+\varepsilon$.

Since $\varepsilon$ is arbitrary, we obtain the claim, and the proof is complete.

Corollary 4.4. Let $\Phi$ be an $N$-function. Let $\left(K_{i}\right)_{i \geq 1}$ be a decreasing sequence of compact sets in $X$ and let $K=\bigcap_{i=1}^{\infty} K_{i}$. Then

$$
C_{\Phi, \mu}(K)=\lim _{i \rightarrow \infty} C_{\Phi, \mu}\left(K_{i}\right) .
$$

Proof. This is a direct consequence of the fact that $C_{\Phi, \mu}$ is an outer capacity.

Theorem 4.5. Let $\Phi$ be a uniformly convex $N$-function such that $\Phi$ satisfies the $\Delta_{2}$ condition. Let $\left(O_{i}\right)_{i \geq 1}$ be an increasing sequence of open sets in $X$ and let $O=$ $\bigcup_{i=1}^{\infty} O_{i}$. Then

$$
C_{\Phi, \mu}(O)=\lim _{i \rightarrow \infty} C_{\Phi, \mu}\left(O_{i}\right)
$$

Proof. The hypothesis implies that $\mathbf{L}_{\Phi, \mu}(X)$ is uniformly convex. By monotonicity, $\lim _{i \rightarrow \infty} C_{\Phi, \mu}\left(O_{i}\right) \leq C_{\Phi, \mu}(O)$. To prove the reverse inequality, we may assume that $\lim _{i \rightarrow \infty} C_{\Phi, \mu}\left(O_{i}\right)<\infty$. Let $\varepsilon>0$ and for $i=1,2, \ldots, u_{i} \in B\left(O_{i}\right)$, and $g_{u_{i}} \in$ $D\left(u_{i}\right) \cap \mathbf{L}_{\Phi, \mu}(X)$ be such that

$$
\left\|\left|u_{i}\right|\right\|_{\Phi, \mu, X}+\left\|\left|g_{u_{i}}\right|\right\|_{\Phi, \mu, X} \leq C_{\Phi, \mu}\left(O_{i}\right)+\varepsilon
$$

The sequence $\left(u_{i}\right)_{i}$ is bounded in $\mathbf{L}_{\Phi, \mu}(X)$ and, hence, it possesses a weakly convergent subsequence, which we denote again by $\left(u_{i}\right)_{i}$. The sequence $\left(g_{u_{i}}\right)_{i}$ is also bounded in $\mathbf{L}_{\Phi, \mu}(X)$ and, by passing to a subsequence, we may assume that $u_{i} \rightarrow u$ weakly in $\mathbf{L}_{\Phi, \mu}(X)$ and $g_{u_{i}} \rightarrow g$ weakly in $\mathbf{L}_{\Phi, \mu}(X)$. We use the Banach-Saks theorem to deduce that the sequence defined by $v_{j}=j^{-1} \sum_{i=1}^{j} u_{i}$ converges to $u$ in $\mathbf{L}_{\Phi, \mu}(X)$ and $g_{v_{j}}=j^{-1} \sum_{i=1}^{j} g_{u_{i}}$ converges to $g$ in $\mathbf{L}_{\Phi, \mu}(X)$.

Now there is a subsequence of the sequence $\left(v_{j}\right)_{j}$ so that $v_{j} \rightarrow u \mu$-a.e. and $g_{v_{j}} \rightarrow g \mu$-a.e. By Lemma 3.7, $u \in M_{\Phi, \mu}^{1}(X)$. On the other hand, $v_{j} \rightarrow 1 \mu$-a.e. in $O$ and hence $u \geq 1 \mu$-a.e. in $O$. Thus $u \in B(O)$. By the weak lower semicontinuity of norms, we get

$$
\begin{aligned}
C_{\Phi, \mu}(O) & \leq\||u|\|_{\Phi, \mu, X}+\||g|\|_{\Phi, \mu, X} \\
& \leq \liminf _{i \rightarrow \infty}\left(\left\|\left|u_{i}\right|\right\|\left\|_{\Phi, \mu, X}+\right\|\left|g_{u_{i}}\right| \|_{\Phi, \mu, X}\right) \\
& \leq \lim _{i \rightarrow \infty} C_{\Phi, \mu}\left(O_{i}\right)+\varepsilon .
\end{aligned}
$$

By letting $\varepsilon \rightarrow 0$, we obtain the result.

The set function $C_{\Phi, \mu}$ is called the $\Phi$-capacity. If a statement holds except on a set $E$ where $C_{\Phi, \mu}(E)=0$, then we say that the statement holds $\Phi$-quasieverywhere (abbreviated $\Phi$-q.e.). 
LemmA 4.6. Let $\Phi$ be any $N$-function and let $u$ be a function in $M_{\Phi, \mu}^{1}(X)$. If $\mathrm{PL}_{u}=$ $\left\{x \in X: \lim _{t \rightarrow x} u(t)=\infty\right\}$ denotes the set of poles of $u$, then $C_{\Phi, \mu}\left(\mathrm{PL}_{u}\right)=0$.

Proof. If $n$ is any positive integer, the function $u_{n}=n^{-1} \inf (u, n)$ is an admissible function for the $\Phi$-capacity of the set $\mathrm{PL}_{u}$. It is easily seen that $\left\|\left|u_{n}\right|\right\|_{M_{\Phi, \mu}^{1}(X)} \leq$ $n^{-1}\||u|\|_{M_{\Phi, \mu}^{1}(X)}$. This implies that $\lim _{n \rightarrow \infty}\left\|\left|u_{n}\right|\right\|_{M_{\Phi, \mu}^{1}(X)}=0$. Thus $C_{\Phi, \mu}\left(\mathrm{PL}_{u}\right)=0$.

Theorem 4.7. Let $\Phi$ be an $N$-function and let $E$ be any subset of $X$. Then $C_{\Phi, \mu}(E)=0$ if and only if for any $\varepsilon>0$, there exists a nonnegative function $u \in$ $M_{\Phi, \mu}^{1}(X)$ such that $\lim _{t \rightarrow x} u(t)=\infty$ for any $x \in E$ and $\||u|\|_{M_{\Phi, \mu}^{1}(X)} \leq \varepsilon$.

Proof. Let $E \subset X$ be such that $C_{\Phi, \mu}(E)=0$, and let $\varepsilon>0$. Then, by the definition of the $\Phi$-capacity, there is a sequence of nonnegative functions, $\left(u_{n}\right)_{n}$, such that $u_{n}=1$ in some neighborhood of $E$ and $\left\|\left|u_{n}\right|\right\|_{M_{\Phi, \mu}^{1}(X)} \leq 2^{-n} \varepsilon$, for any $n$. Then the function $u=\sum_{n} u_{n}$ belongs to $M_{\Phi, \mu}^{1}(X)$ and $\lim _{t \rightarrow x} u(t)=\infty$. Furthermore, it is evident that $\||u|\|_{M_{\Phi, \mu}^{1}(X)} \leq \varepsilon$.

The converse implication is an immediate consequence of Lemma 4.6.

Theorem 4.8. Let $\Phi$ be an N-function and $\left(u_{i}\right)_{i}$ be a Cauchy sequence of functions in $M_{\Phi, \mu}^{1}(X) \cap \mathbf{C}(X)$. Then, there is a subsequence $\left(u_{i}^{\prime}\right)_{i}$ of $\left(u_{i}\right)_{i}$ which converges pointwise $\Phi$-q.e. in $X$. Moreover, the convergence is uniform outside a set of arbitrary small $\Phi$-capacity.

Proof. Since $\left(u_{i}\right)_{i}$ is a Cauchy sequence, there is a subsequence $\left(u_{i}^{\prime}\right)_{i}$ of $\left(u_{i}\right)_{i}$ such that

$$
\sum_{i=1}^{\infty} 2^{i}\left\|\left|u_{i}^{\prime}-u_{i+1}^{\prime}\right|\right\|_{M_{\Phi, \mu}^{1}(X)}<\infty
$$

We set $X_{i}=\left\{x \in X:\left|u_{i}^{\prime}(x)-u_{i+1}^{\prime}(x)\right|>2^{-i}\right\}$ for $i=1,2, \ldots$, and $Y_{j}=\bigcup_{i=j}^{\infty} X_{i}$. Since the functions $u_{i}^{\prime}$ are continuous by hypothesis, $X_{i}$ and $Y_{j}$ are open. Then, for all $i, 2^{i}\left(u_{i}^{\prime}-u_{i+1}^{\prime}\right) \in B\left(X_{i}\right)$. Hence

$$
C_{\Phi, \mu}\left(X_{i}\right) \leq 2^{i}\left\|\left|u_{i}^{\prime}-u_{i+1}^{\prime}\right|\right\|_{M_{\Phi, \mu}^{1}(X)} .
$$

By subadditivity of $C_{\Phi, \mu}$, we obtain

$$
C_{\Phi, \mu}\left(Y_{j}\right) \leq \sum_{i=j}^{\infty} C_{\Phi, \mu}\left(X_{i}\right) \leq \sum_{i=j}^{\infty} 2^{i}\left\|\left|u_{i}^{\prime}-u_{i+1}^{\prime}\right|\right\|_{M_{\Phi, \mu}^{1}(X)} .
$$

From the convergence of the sum (4.12) we conclude that

$$
C_{\Phi, \mu}\left(\bigcap_{j=1}^{\infty} Y_{j}\right) \leq \lim _{j \rightarrow \infty} C_{\Phi, \mu}\left(Y_{j}\right)=0
$$


Thus $\left(u_{i}^{\prime}\right)_{i}$ converges in $X \backslash \bigcap_{j=1}^{\infty} Y_{j}$. Moreover, we have for any $x \in X \backslash Y_{j}$ and all $k>j$

$$
\left|u_{j}^{\prime}(x)-u_{k}^{\prime}(x)\right| \leq \sum_{i=j}^{k-1}\left|u_{i}^{\prime}(x)-u_{i+1}^{\prime}(x)\right| \leq \sum_{i=j}^{k-1} 2^{-i} \leq 2^{1-j} .
$$

This implies that $\left(u_{i}^{\prime}\right)_{i}$ converges uniformly in $X \backslash Y_{j}$.

This completes the proof.

Definition 4.9. Let $\Phi$ be an $N$-function. A function $u: X \rightarrow[-\infty,+\infty]$ is $\Phi$ quasi-continuous in $X$ if for every $\varepsilon>0$, there is a set $E$ such that $C_{\Phi, \mu}(E)<\varepsilon$ and the restriction of $u$ to $X \backslash E$ is continuous. Since $C_{\Phi, \mu}$ is an outer capacity, we may assume that $E$ is open.

Theorem 4.10. Let $\Phi$ be an $N$-function satisfying the $\Delta_{2}$ condition and $u \in$ $M_{\Phi, \mu}^{1}(X)$. Then there is a function $v \in M_{\Phi, \mu}^{1}(X)$ such that $u=v \mu$-a.e. and $v$ is $\Phi$-quasi-continuous in $X$. The function $v$ is called a $\Phi$-quasi-continuous representative of $u$.

Proof. We know that $M_{\Phi, \mu}^{1}(X)$ is a Banach space and by Theorem 3.10, $\mathrm{C}(X) \cap$ $M_{\Phi, \mu}^{1}(X)$ is a dense subspace of $M_{\Phi, \mu}^{1}(X)$. Hence, completeness implies that $M_{\Phi, \mu}^{1}(X)$ can be characterized as the completion of $\mathbf{C}(X) \cap M_{\Phi, \mu}^{1}(X)$ in the norm of $M_{\Phi, \mu}^{1}(X)$. Thus there are sequences of functions $\left(u_{i}\right)_{i} \subset \mathbf{C}(X) \cap \mathbf{L}_{\Phi, \mu}(X)$ and $\left(g_{i}\right)_{i} \subset D\left(u_{i}-u\right)$ such that $u_{i} \rightarrow u$ and $g_{i} \rightarrow 0$ in $\mathbf{L}_{\Phi, \mu}(X)$. Hence for a subsequence, which we denote again by $\left(u_{i}\right)_{i}, u_{i} \rightarrow u \mu$-a.e. From Theorem 4.8 , we deduce that the limit function $v$ of the sequence $\left(u_{i}\right)_{i}$ is $\Phi$-quasi-continuous in $X$. This completes the proof.

\section{Comparison between capacity and measures}

5.1. Comparison between capacity and the measure $\mu$. The sets of zero capacity are exceptional sets in the strongly nonlinear potential theory. We show in the next lemma that sets of vanishing capacity are also of measure zero.

Lemma 5.1. Let $\Phi$ be any $N$-function. Then

(1) if $E \subset X$ is such that $C_{\Phi, \mu}(E)=0$, then $\mu(E)=0$;

(2) if $E \subset X$ is such that $\mu(E) \neq 0$, then $C_{\Phi, \mu}(E) \geq 1 / \Phi^{-1}(1 / \mu(E))$.

Proof. (1) If $n$ is a strictly positive integer, we can find $u_{n} \in B(E)$ such that $\left\|\left|n u_{n}\right|\right\|_{\Phi, \mu, X} \leq\left\|\left|n u_{n}\right|\right\|_{M_{\Phi, \mu}^{1}(X)} \leq 1$. Hence, there is an open set $O_{n}$ such that $E \subset$ $O_{n}$ and $u_{n} \geq 1$ in $O_{n}$. Then

$$
\int_{E} \Phi\left(n u_{n}\right) d \mu \leq \int_{O_{n}} \Phi\left(n u_{n}\right) d \mu \leq \int_{X} \Phi\left(n u_{n}\right) d \mu \leq 1 .
$$

By the inequality $n \Phi\left(u_{n}\right) \leq \Phi\left(n u_{n}\right)$ and by the fact that $u_{n} \geq 1$ in $E$, we get $\Phi(1) \mu(E) \leq 1 / n$. This implies the claim. 
(2) Let $u \in B(E)$, then there is an open set $O$ such that $E \subset O$ and $u \geq 1$ in $O$. Hence

$$
\int_{X} \Phi\left[u \cdot \Phi^{-1}\left(\frac{1}{\mu(E)}\right)\right] d \mu \geq \int_{E} \Phi\left[u \cdot \Phi^{-1}\left(\frac{1}{\mu(E)}\right)\right] d \mu \geq 1
$$

Thus

$$
\||u|\|_{M_{\Phi, \mu}^{1}(X)} \geq\left.\||u|\|\right|_{\Phi, \mu, X} \geq \frac{1}{\Phi^{-1}(1 / \mu(E))} .
$$

We obtain the result by taking the infimum over all $u \in B(E)$.

Definition 5.2. A measure $\mu$ is said to be doubling if there is a constant $C \geq 1$ such that

$$
\mu(B(x, 2 r)) \leq C \mu(B(x, r))
$$

for every $x \in X$ and $r>0$.

The smallest constant $C$ in (5.4) is called the doubling constant and is denoted by $C_{d}$.

Theorem 5.3. Let $\Phi$ be any $N$-function, $\mu$ a doubling measure with the doubling constant $C_{d}, x_{0} \in X$, and $0<r \leq 1$. Then

$$
C_{\Phi, \mu}\left(B\left(x_{0}, r\right)\right) \leq 2 C_{d} r^{-1} \frac{1}{\Phi^{-1}\left(1 / \mu\left(B\left(x_{0}, r\right)\right)\right)} .
$$

Proof. We define

$$
u(x)= \begin{cases}\frac{2 r-d\left(x, x_{0}\right)}{r}, & x \in B\left(x_{0}, 2 r\right) \backslash B\left(x_{0}, r\right), \\ 1, & x \in B\left(x_{0}, r\right), \\ 0, & x \in X \backslash B\left(x_{0}, 2 r\right) .\end{cases}
$$

Define also

$$
g(x)= \begin{cases}\frac{1}{r}, & x \in B\left(x_{0}, 2 r\right), \\ 0, & x \in X \backslash B\left(x_{0}, 2 r\right) .\end{cases}
$$

We show that $g \in D(u)$. Let $E=B\left(x_{0}, 2 r\right) \backslash B\left(x_{0}, r\right)$, and $x, y \in E$. Then

$$
|u(x)-u(y)|=\frac{\left|d\left(x, x_{0}\right)-d\left(y, x_{0}\right)\right|}{r} \leq \frac{d(x, y)}{r} .
$$

Hence (3.12) follows in this case. Let $x \in E$ and $y \in B\left(x_{0}, r\right)$. We have

$$
|u(x)-u(y)|=1-u(x)=\frac{d\left(x, x_{0}\right)-r}{r}
$$


Since $d\left(y, x_{0}\right)<r \leq d\left(x, x_{0}\right)$, we get

$$
d\left(x, x_{0}\right)-r \leq d\left(x, x_{0}\right)-d\left(y, x_{0}\right) \leq d(x, y)
$$

and hence (3.12) follows. The case $y \in E$ and $x \in B\left(x_{0}, r\right)$ is completely analogous. Now, if $x, y \in B\left(x_{0}, r\right)$ or $x, y \in X \backslash B\left(x_{0}, 2 r\right)$, then clearly (3.12) holds. On the other hand, let $x \in X \backslash B\left(x_{0}, 2 r\right)$ and $y \in B\left(x_{0}, r\right)$. Then

$$
|u(x)-u(y)|=1 \leq \frac{d(x, y)}{r}
$$

which implies (3.12). Finally, if $x \in E$ and $y \in X \backslash B\left(x_{0}, 2 r\right)$, then

$$
|u(x)-u(y)|=u(x)=\frac{2 r-d\left(x, x_{0}\right)}{r}
$$

and since $d\left(x, x_{0}\right)<2 r<d\left(y, x_{0}\right)$, we obtain

$$
2 r-d\left(x, x_{0}\right) \leq d\left(y, x_{0}\right)-d\left(x, x_{0}\right) \leq d(x, y)
$$

and (3.12) holds. Thus $g \in D(u), u \in B\left(B\left(x_{0}, r\right)\right)$ and

$$
C_{\Phi, \mu}\left(B\left(x_{0}, r\right)\right) \leq\left\||| u\left|\left\|\left.\right|_{\Phi, \mu, X}+\right\|\right| g \mid\right\|_{\Phi, \mu, X} \cdot
$$

We have $u \leq \chi_{B\left(x_{0}, 2 r\right)},\||u|\|_{\Phi, \mu, X} \leq\left\|\left|\chi_{B\left(x_{0}, 2 r\right)}\right|\right\|_{\Phi, \mu, X}$. We know that

$$
\|\left.\right|_{B\left(x_{0}, 2 r\right)}||_{\Phi, \mu, B\left(x_{0}, 2 r\right)}=\frac{1}{\Phi^{-1}\left(1 / \mu\left(B\left(x_{0}, 2 r\right)\right)\right)},
$$

where $\chi_{B\left(x_{0}, 2 r\right)}$ is the characteristic function of $B\left(x_{0}, 2 r\right)$.

On the other hand, $g \leq r^{-1} \chi_{B\left(x_{0}, 2 r\right)}$, and hence

$$
\||g|\|_{\Phi, \mu, X} \leq r^{-1} \frac{1}{\Phi^{-1}\left(1 / \mu\left(B\left(x_{0}, 2 r\right)\right)\right)} .
$$

Thus

$$
C_{\Phi, \mu}\left(B\left(x_{0}, r\right)\right) \leq\left(1+r^{-1}\right) \frac{1}{\Phi^{-1}\left(1 / \mu\left(B\left(x_{0}, 2 r\right)\right)\right)} .
$$

Now, since $\mu$ is doubling, $\mu\left(B\left(x_{0}, 2 r\right)\right) \leq C_{d} \mu\left(B\left(x_{0}, r\right)\right)$. Hence

$$
\frac{1}{\Phi^{-1}\left(1 / \mu\left(B\left(x_{0}, 2 r\right)\right)\right)} \leq \frac{1}{\Phi^{-1}\left(1 / C_{d} \mu\left(B\left(x_{0}, r\right)\right)\right)}
$$


372 Strongly nonlinear potential theory on metric spaces

It remains to evaluate $\Phi^{-1}\left(1 / C_{d} \mu\left(B\left(x_{0}, r\right)\right)\right)$. Recall that, $\Phi(\alpha x) \leq \alpha \Phi(x)$, for all $0 \leq \alpha \leq 1$, and all $x$. This implies that $\alpha \Phi^{-1}(x) \leq \Phi^{-1}(\alpha x)$, for all $0 \leq \alpha \leq 1$, and all $x$. Hence

$$
\frac{1}{\Phi^{-1}\left(1 / C_{d} \mu\left(B\left(x_{0}, r\right)\right)\right)} \leq C_{d} \frac{1}{\Phi^{-1}\left(1 / \mu\left(B\left(x_{0}, r\right)\right)\right)} .
$$

Thus

$$
\begin{aligned}
C_{\Phi, \mu}\left(B\left(x_{0}, r\right)\right) & \leq\left(1+r^{-1}\right) C_{d} \frac{1}{\Phi^{-1}\left(1 / \mu\left(B\left(x_{0}, r\right)\right)\right)} \\
& \leq 2 r^{-1} C_{d} \frac{1}{\Phi^{-1}\left(1 / \mu\left(B\left(x_{0}, r\right)\right)\right)} .
\end{aligned}
$$

This completes the proof.

5.2. Comparison between capacity and Hausdorff measures. We recall the definition of Hausdorff measures. Let $h:[0,+\infty[\rightarrow[0,+\infty[$ be an increasing function such that $\lim _{r \rightarrow 0} h(r)=0$. For $0<\delta \leq \infty$ and $E \subset X$, we define

$$
\mathbf{H}_{\delta}^{h}(E)=\inf \left\{\sum_{i=1}^{\infty} h\left(r_{i}\right): E \subset \bigcup_{i=1}^{\infty} B\left(x_{i}, r_{i}\right), r_{i} \leq \delta\right\} .
$$

The $h$-Hausdorff measure of $E$ is given by

$$
\mathbf{H}^{h}(E)=\lim _{\delta \rightarrow 0} \mathbf{H}_{\delta}^{h}(E) .
$$

If $h(t)=t^{s}$ for $0 \leq s<\infty$, we obtain the $s$-dimensional Hausdorff measure which we denote by $\mathbf{H}^{s}$.

For more details on Hausdorff measures, one can consult [8, Chapter 2, Section 10].

A measure $\mu$ is regular with dimension $s>0$, if there is $c \geq 1$ such that, for each $x \in X$ and $0<r \leq \operatorname{diam}(X)$,

$$
c^{-1} r^{s} \leq \mu(B(x, r)) \leq c r^{s} .
$$

Note that if $\mu$ is regular with dimension $s$, then $\mu$ is doubling. Moreover, $X$ has Hausdorff dimension $s$ and there is a constant $c>0$ such that, for every $E \subset X$,

$$
c^{-1} \mathbf{H}^{s}(E) \leq \mu(E) \leq c \mathbf{H}^{s}(E) .
$$

Theorem 5.4. Let $\Phi$ be an $N$-function satisfying the $\Delta_{2}$ condition and $\alpha=\alpha(\Phi)=$ $\sup _{t>0}(t \varphi(t) / \Phi(t))$. Let $\mu$ be a regular measure with dimension $s$ such that $s>\alpha$. Define $h:\left[0,+\infty\left[\rightarrow\left[0,+\infty\left[\right.\right.\right.\right.$ by $h(t)=t^{s / \alpha-1}$. Then for every $E \subset X$,

$$
C_{\Phi, \mu}(E) \leq c \mathbf{H}^{h}(E)
$$


where the constant $c$ depends only on $\Phi$ and the doubling constant. In particular, if $\mathbf{H}^{h}(E)=0$, then $C_{\Phi, \mu}(E)=0$.

Proof. Let $B\left(x_{i}, r_{i}\right), i=1,2, \ldots$, be any covering of $E$ such that the radii $r_{i}$ satisfy $r_{i} \leq 1 / 2, i=1,2, \ldots$ By $(5.5)$ we get, for all $i$, that

$$
C_{\Phi, \mu}\left(B\left(x_{i}, r_{i}\right)\right) \leq C r^{-1} \frac{1}{\Phi^{-1}\left(1 / \mu\left(B\left(x_{i}, r_{i}\right)\right)\right)} .
$$

From (5.23) we have $\mu\left(B\left(x_{i}, r_{i}\right)\right) \leq c r_{i}^{s}$, for all $i$; and hence

$$
\Phi^{-1}\left(\frac{1}{\mu\left(B\left(x_{i}, r_{i}\right)\right)}\right) \geq \Phi^{-1}\left(\frac{1}{c r_{i}^{s}}\right) \geq c^{-1} \Phi^{-1}\left(\frac{1}{r_{i}^{s}}\right) .
$$

It remains to evaluate $\Phi^{-1}\left(1 / r_{i}^{s}\right)$. For this goal we distinguish two cases

(i) if $\Phi(1) \leq 1$, then $\Phi^{-1}\left(1 / r_{i}^{s}\right) \geq \Phi^{-1}\left(\Phi(1)\left(1 / r_{i}^{s}\right)\right)$;

(ii) if $\Phi(1) \geq 1$, then

$$
\Phi^{-1}\left(\frac{1}{r_{i}^{s}}\right)=\Phi^{-1}\left(\frac{\Phi(1)}{\Phi(1) r_{i}^{s}}\right) \geq \frac{1}{\Phi(1)} \Phi^{-1}\left(\Phi(1) \frac{1}{r_{i}^{s}}\right)
$$

Hence in the two cases we have $\Phi^{-1}\left(1 / r_{i}^{s}\right) \geq C^{\prime} \Phi^{-1}\left(\Phi(1)\left(1 / r_{i}^{s}\right)\right)$. We get

$$
\Phi^{-1}\left(\frac{1}{\mu\left(B\left(x_{i}, r_{i}\right)\right)}\right) \geq C^{\prime} \Phi^{-1}\left(\Phi(1) \frac{1}{r_{i}^{s}}\right)
$$

On the other hand we know that $\Phi(t) \leq \Phi(1) t^{\alpha}$, for all $t \geq 1$. This implies that $t^{1 / \alpha} \leq \Phi^{-1}(\Phi(1) t)$, for all $t \geq 1$. Whence, for all $i$

$$
\Phi^{-1}\left(\frac{1}{\mu\left(B\left(x_{i}, r_{i}\right)\right)}\right) \geq C^{\prime} \frac{1}{r_{i}^{s / \alpha}}
$$

Thus, for all $i$

$$
C_{\Phi, \mu}\left(B\left(x_{i}, r_{i}\right)\right) \leq C^{\prime \prime} r^{s / \alpha-1}
$$

The subadditivity of $C_{\Phi, \mu}$ yields

$$
C_{\Phi, \mu}(E) \leq C^{\prime \prime} \sum_{i=1}^{\infty} C_{\Phi, \mu}\left(B\left(x_{i}, r_{i}\right)\right) \leq C^{\prime \prime} \sum_{i=1}^{\infty} h\left(r_{i}\right) .
$$

By taking the infimum over all coverings by balls and letting the radii tend to zero, we finish the proof. 


\section{References}

[1] R. A. Adams, Sobolev Spaces, Academic Press, New York, 1975.

[2] N. Aïssaoui, Note sur la capacitabilité dans les espaces d'Orlicz, Ann. Sci. Math. Québec 19 (1995), no. 2, 107-113 (French).

[3] , Bessel potentials in Orlicz spaces, Rev. Mat. Univ. Complut. Madrid 10 (1997), no. 1, 55-79.

[4] Instability of capacity in Orlicz spaces, Potential Anal. 6 (1997), no. 4, 327346.

[5] Some developments of strongly nonlinear potential theory, Libertas Math. 19 (1999), 155-170.

[6] N. Aïssaoui and A. Benkirane, Capacités dans les espaces d'Orlicz, Ann. Sci. Math. Québec 18 (1994), no. 1, 1-23 (French).

[7] Potentiel non linéaire dans les espaces d'Orlicz, Ann. Sci. Math. Québec 18 (1994), no. 2, 105-118 (French).

[8] H. Federrer, Geometric Measure Theory, Springer-Verlag, Berlin, 1969.

[9] B. Franchi, P. Hajłasz, and P. Koskela, Definitions of Sobolev classes on metric spaces, Ann. Inst. Fourier (Grenoble) 49 (1999), no. 6, 1903-1924.

[10] D. Gallardo, Orlicz spaces for which the Hardy-Littlewood maximal operator is bounded, Publ. Mat. 32 (1988), no. 2, 261-266.

[11] D. Gilbarg and N. S. Trudinger, Elliptic Partial Differential Equations of Second Order, Springer-Verlag, Berlin, 1983.

[12] P. Hajłasz, Sobolev spaces on an arbitrary metric space, Potential Anal. 5 (1996), no. 4, 403-415.

[13] P. Hajłasz and P. Koskela, Sobolev met Poincaré, Mem. Amer. Math. Soc. 145 (2000), no. $688, \mathrm{x}+101$.

[14] P. Hajłasz and O. Martio, Traces of Sobolev functions on fractal type sets and characterization of extension domains, J. Funct. Anal. 143 (1997), no. 1, 221-246.

[15] L. I. Hedberg, On certain convolution inequalities, Proc. Amer. Math. Soc. 36 (1972), 505-510.

[16] T. Kilpeläinen, J. Kinnunen, and O. Martio, Sobolev spaces with zero boundary values on metric spaces, Potential Anal. 12 (2000), no. 3, 233-247.

[17] J. Kinnunen and O. Martio, The Sobolev capacity on metric spaces, Ann. Acad. Sci. Fenn. Math. 21 (1996), no. 2, 367-382.

[18] M. A. Krasnosel'skiı̌ and Ja. B. Rutickiü, Convex Functions and Orlicz Spaces, P. Noordhoff, Groningen, 1961.

[19] A. Kufner, O. John, and S. Fučík, Function Spaces, Noordhoff International Publishing, Leyden, 1977.

[20] W. A. J. Luxemburg, Banach Function Spaces, Thesis, Technische Hogeschool te Delft, 1955.

[21] M. M. Rao and Z. D. Ren, Theory of Orlicz Spaces, Marcel Dekker, New York, 1991.

[22] L. Simon, Lectures on Geometric Measure Theory, Australian National University Centre for Mathematical Analysis, Canberra, 1983.

[23] W. P. Ziemer, Weakly Differentiable Functions, Springer-Verlag, New York, 1989.

Noureddine Aïssaoui: École Normale Supérieure, B.P. 5206, Ben Souda, Fès, Morocco

E-mail address: n.aissaoui@caramail.com 


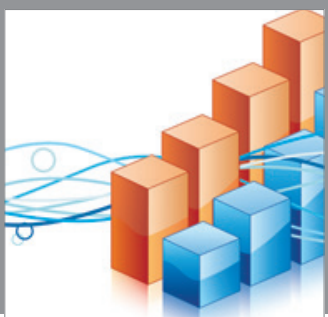

Advances in

Operations Research

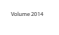

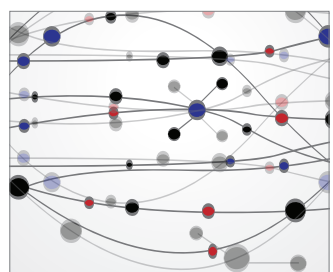

\section{The Scientific} World Journal
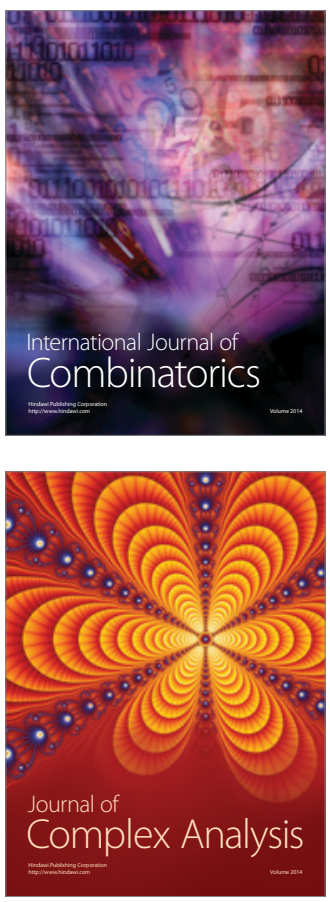

International Journal of

Mathematics and

Mathematical

Sciences
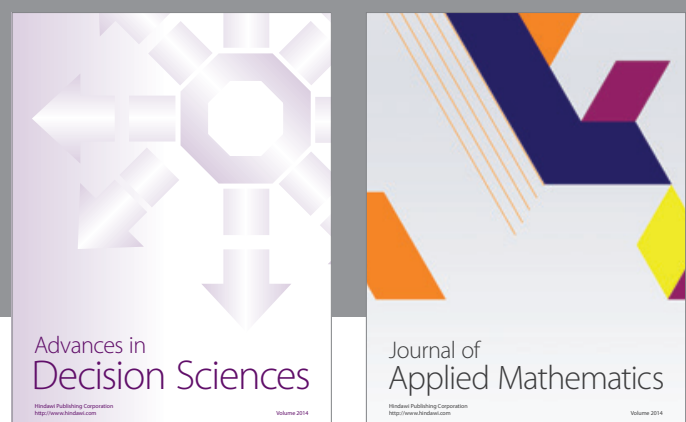

Journal of

Applied Mathematics
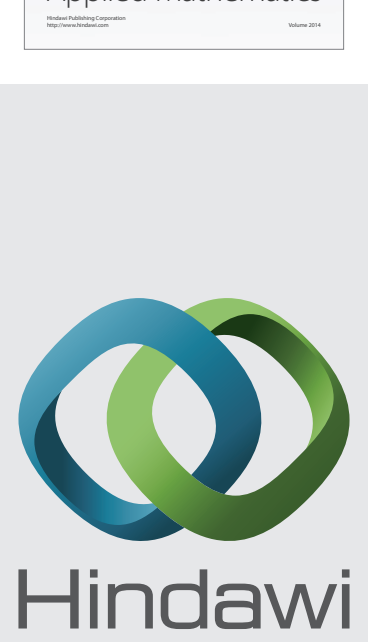

Submit your manuscripts at http://www.hindawi.com
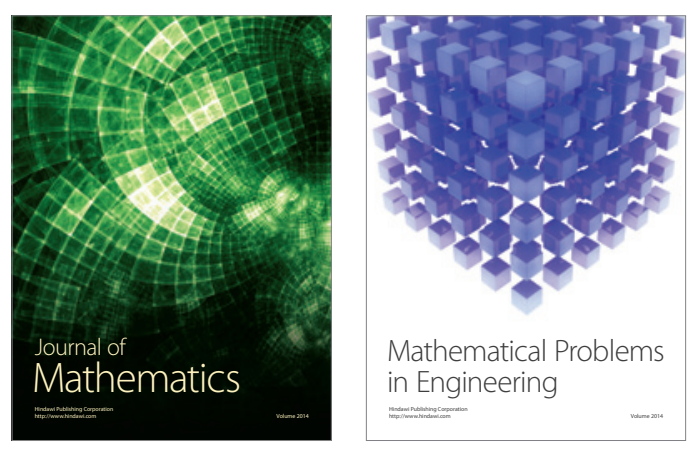

Mathematical Problems in Engineering
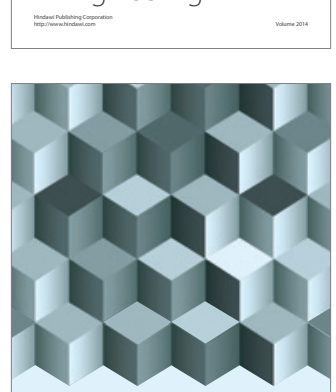

Journal of

Function Spaces
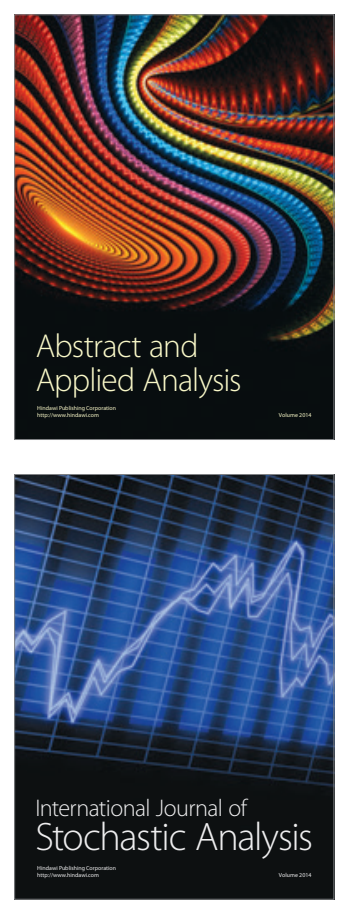

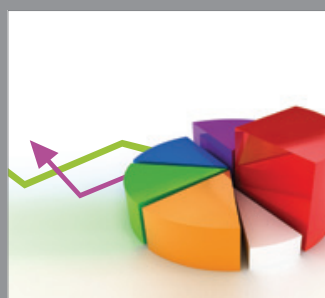

ournal of

Probability and Statistics

Promensencen
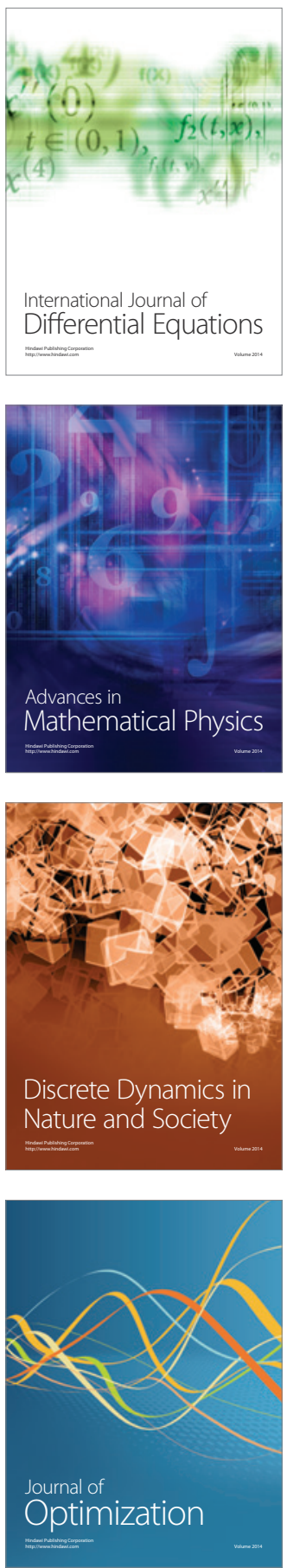